\title{
POSTERS
}

\section{Vertical Abundances Variation in the Galaxy: The Case of PN}

\author{
F. Cuisinier ${ }^{1,2}$ and J.Köppen ${ }^{2}$ \\ ${ }^{1}$ IAG/USP, São Paulo, Brazil; ${ }^{2}$ Obs. Strasbourg, France
}

The vertical structure of the Galaxy is stratified in different populations. Gas and young stars have a low velocity dispersion (about $10 \mathrm{~km} / \mathrm{s}$ ) and thus are confined in a very thin disk (100 pc of scale height). Old stars have larger velocity dispersions, and are thus encountered up to a few kpc.

PN can be observed at very large distances in the Galaxy, because the whole spectrum is concentrated in a few lines of emission. Thus, the chemical composition of the interstellar medium where the progenitor was born can be determined. The stellar life times cover a wide range: roughly from $1 \mathrm{Gyr}$ to $15 \mathrm{Gyr}$. The massive $\left(M_{*}>3 M_{\odot}\right.$, short-lived stars produce nebulae enriched in helium and in nitrogen - the products of the stellar nucleosynthesis (Type I). Most nebulae however come from less massive stars, with long life times, which make PN very useful as probes into the history of galactic evolution.

Thus, studies with PN allow to test the results of stellar studies, limited to the solar neighbourhood, over a larger portion of the galactic disk.

From a sample of PN with high quality spectra we define a group of 94 objects that belong to the galactic disk, and may therefore represent the solar neighbourhood. Among these objects, we find a systematic decrease of the abundances with the height above the plane. For $\mathrm{O}, \mathrm{S}, \mathrm{Ar}$, this amounts to $\simeq 0.1-0.2$ dex between $\mathrm{z}=0$ and $\mathrm{z}=3 \mathrm{kpc}$.

We build a simple model for the chemical and kinematical evolution of the solar neighbourhood, based on stellar data. We take as well into account the radial diffusion of stellar orbits and we simulate the influence from the uncertainties of the nebular distances. The model predicts an abundance variation of $0.1-0.2$ dex, in good accord with the observations.

We conclude that the 'classical' model of the evolution of the stellar disk by some form of dynamical heating of the stars in conjunction with our empirical understanding of the chemical evolution in the solar neighbourhood is consistent with the data obtained from the planetary nebulae on the metallicity structure in the galactic disk. 